\title{
The Voltage Regulation Control Strategy with Distributed Photovoltaic Participation under Serious Failure Based on Transient Voltage Margin
}

\author{
Yunxin Liu ${ }^{1}$, Liangzhong $\mathrm{Yao}^{1}$, Siyang $\mathrm{Liao}^{1}$, Jian $\mathrm{Xu}^{1}, D i \mathrm{Wu}^{2}$, Wei $\mathrm{Tang}^{2}$ and Cheng Yang $^{2}$ \\ ${ }^{1}$ the School of Electrical Engineering and Automation, Wuhan University, Wuhan, China \\ 2 the State Grid, Anhui Provincial Electric Power Company, Anhui, China
}

\begin{abstract}
With the poor ability of distributed PV to withstand voltage fluctuation, the voltage fluctuation caused by DC blocking can lead to large-scale disorderly de-networking of distributed PV, especially those distributed PV which close to voltage weak buses. which means that more and more attentions are paid to the transient voltage stability (TVS). If distributed PV can participation in reactive compensation, we don't need to add additional reactive power compensator, voltage stability of voltage weak buses will be increased, which can reduce PV off-grid. Therefore, for the HVDC receiving end system under distributed PV intensive access, first of all, we need to find the voltage weak bus, then increase the reactive power output of distributed PV near the voltage weak buses to increase transient voltage margin. Taking the simplified model of power system in Anhui Province as an example, the validity of the strategy is verified, and the results show that the transient voltage stability of the system has been improved.
\end{abstract}

\section{Introduction}

The power of the DC transmission system is fed into the receiving end system in large numbers, resulting in a reduction in the dynamic reactive power supply of the receiving end system and a decrease in voltage support capacity. Under the low voltage state after the system disturbance, the dynamic response characteristics of DC converter and induction motor load will consume a lot of reactive power, further reduce the system reactive power reserve, and the temporary voltage stability problem of the end system is prominent (see reference [1-2] ). And after the distributed PV is densely connected to the UHVDC receiving end system, the distributed PV replaces the conventional power supply, which reduces the voltage supporting ability and short-circuit capacity to a certain extent, so the voltage regulation capacity is weakened. What's more, when the DC is blocked, a short-term local reactive power excess will occur near the converter station, resulting in a sharp rise in the bus voltage near the converter station. After that, the voltage will gradually decrease with the gradual removal of the reactive power compensation equipment. With the poor ability of distributed PV to withstand voltage fluctuation, the voltage fluctuation caused by DC blocking can lead to large-scale disorderly de-networking of distributed PV, especially those distributed PV which close to voltage weak buses. In addition, the large-scale power flow transfer of the receiving end system will also cause some transmission lines to exceed limits and cause some nodes to have lower voltages. If not handled in time, it may also trigger a chain reaction, further expand the scope of the accident, increase the severity of the accident, and even cause a large-scale voltage collapse and power outage (see reference [3-4] ). Therefore, for the HVDC receiving end system under distributed PV intensive access, the system stability is more complicated, and the issue of transient voltage stability deserves more attention.

Many scholars have made researches on the transient voltage stability of DC receiving end system. Reference[5] takes Hunan province power grid of Qilian-Shaoshan DC system's receiving-end as an example, together with Shaoshan synchronous condenser project and protective system construction of Central China power grid, and builds a coordinated multi-objective reactive voltage control system to make full use of the reactive power resources of synchronous condensers, LV capacitors and reactors, and generators. Reference [6-8] analyzed the role of the camera in the power grid mainly to provide dynamic reactive power support for the system, improve the voltage stability level in the near area, and suppress transient overvoltages and voltage drops in the power grid. Reference [9-10] proposed the use of measures to install STATCOM devices to achieve dynamic reactive power compensation and achieve the purpose of voltage stabilization. Reference [11] installed a static reactive power compensation device (SVC) at Luodong Station, which has a large dynamic reactive power shortage, it shows the transient voltage stability of the receiving end grid has been greatly improved.

The above methods can significantly improve the transient voltage stability of the receiving end grid, but 
${ }^{a}$ they all need to be equipped with dynamic reactive power compensation devices, which are costly. For the HVDC receiving end system under distributed PV intensive access, the PV inverter has the same topology as SVG and STATCOM, and can also run at any power factor. If PV can provide reactive power support during the transient process, reduce its own grid-connected point voltage fluctuations, prevent itself from being disconnected, and provide reactive power support for weak voltage points(see reference[12-13]), it will save investment in reactive power compensation devices and increase utilization rate of inverter capacity. To this end, this paper proposes a voltage regulation control strategy with distributed photovoltaic participation under serious failure based on transient voltage margin. According to the transient response curve of the receiving end grid after DC blocking, we can define transient voltage margins, and then we can select weak voltage nodes and photovoltaic reactive power control nodes are discussed based on transient voltage margin which are calculated before. Finally, we take Anhui Power Grid as an example, and the proposed voltage regulation control strategy is verified.

\section{Analysis of existing practical criteria and quantitative indicator}

When HVDC is blocked and the reactive power compensation device has not been cut off, the voltage around the point of common coupling of HVDC will first rise suddenly, and the reactive power compensation device in the converter station will be gradually removed After that, the voltage will gradually decrease (see reference [14] ). In this dynamic process, whether the drop of physical quantity (such as voltage, frequency, etc.) is within a given range is an important basis for judging the safety of power system.

Broadly speaking, any method of calculating voltage stabilization indicators can be used to identify the weakness of bus voltage. For a quick and effective determination of voltage stability, there are many practical criteria based on the acceptability of transient voltage drop to judge the stability of the system transient voltage, as described below

\subsection{Practical criteria 1 and derivative indicators}

According to reference [15], the transient voltage safety of power systems includes two aspects: transient voltage acceptability and stability. The voltage binary table $\left(u_{c r}, \tau_{c r}\right)$ is often used to indicate the transient voltage acceptability, which means that the duration of the voltage curve during the transient process should not exceed $u_{c r}$ for a period of time that does not exceed $\tau_{c r}$.

${ }^{\text {a }}$ This work was supported in part by the Science and Technology Projects of State Grid Co. Ltd. China (SGTYHT/18-JS-206).
The system can have multiple voltage binary tables, and determine $u_{c r}$ and $\tau_{c r}$ according to the voltage requirements of electrical equipment in the grid.

In the practical judgment 1, "Technique Specification of Power System Security and Stability Calculation " (see reference [16] ) provides for the temporary voltage stability of the basis as follows: in the power system after the disturbance of the transient process, the load bus voltage can be restored to $0.80 \mathrm{pu}$ or more within $10 \mathrm{~s}$ after the failure.

Based on the above criteria, reference [17] using the integral of the negative load bus voltage below the set value in the transient process, constructs the following evaluation indicators:

$$
\eta_{V i, j}=D_{i, j}+\int_{t}^{t+\Delta t}\left[V_{N}-V_{i, j}(t)\right] d t
$$

$V_{i, j}(t)$ is the voltage of the load bus $j$ when the fault $i$ occurs, $t$ is the moment the fault starts, and $\Delta t$ is the duration of $V_{i, j}(t)$ below the $V_{N}$.

The above indicator $\eta_{V i, j}$ consists of two parts: one is the penalty $D_{i, j}$, the penalty value is 0 when the voltage meets the requirement of the criterion 1 , otherwise the penalty value $p(p \geq 0$, according to different actual system adjustments to clearly distinguish the transient voltage stability and instability situation, reference [18] is based on experience, the value of 10); The second is the integral item, expressed by the area where the bus voltage is lower than $V_{N}$ in the transient process. When $\eta_{V i, j}$ is greater than $p$, the transient voltage of the bus is judged to be instable.

\subsection{Practical criteria 2 and derivative indicators}

In the practical judgment 2, " Guide on security and stability analysis for CSG " (see reference [19] ) provides for the temporary voltage stability of the basis as follows: The voltage drop of the system hub bus continuously below $0.75 \mathrm{pu}$ during the post-failure transient process does not exceed $1 \mathrm{~s}$, and the bus post-bus voltage of $220 \mathrm{kV}$ and above at the end of the transient process is not less than $0.9 \mathrm{pu}$.

Based on the above criteria, reference [20] considers the combined effect of the lowest voltage of the bus transient, the duration of the lower voltage setting, and the recovery voltage of the bus after the failure, and defines the following transient voltage stability margin index before their text citation. If a figure or table is too large to fit into one column, it can be centred across both columns at the top or the bottom of the page.

$$
\eta_{V}=\left\{\begin{array}{l}
V_{\min }-V_{c r}+K_{t}\left(T_{c r}-\frac{T_{b} V_{c r}}{V_{R}}\right), T_{b}<T_{c r} \\
-100 \%, T_{b}<T_{c r}
\end{array}\right.
$$

$V_{c r}$ and $T_{c r}$ are the voltage setting sands and the maximum acceptable duration below the corresponding 
setting, respectively, $V_{\min }$ is the lowest transient voltage of the central bus after fault clearance, $V_{R}$ is the steady state recovery voltage after the fault, $T_{b}$ is the maximum duration of the bus voltage below $V_{c r}$ in the actual fault, and $K_{t}$ is the critical voltage offset time factor.

The indicator improves the judgment 2 , if the judgment is instable, the indicator is set directly to -1 , and if the judgment is stable, the two-part information is weighted to characterize stability. The first is the difference between the minimum temporary voltage and the set voltage. The second is the difference between the critical voltage time and the set time after the steady-state recovery voltage calibration after failure. When $\eta_{V}$ is less than 0 , the transient voltage of the bus is determined to be unstable.

\subsection{More continuous transient voltage stability margin}

In order to quantitatively analyze the transient voltage stability of the system, the reference [21] summed up all the periods when the voltage was lower than the time when the fault was cut to the end of the transient period, and defined the ratio of the accumulated value to the total transient duration as the transient voltage stability margin. The main disadvantage of this definition is the poor smoothness of the index, which is not conducive to optimal search. To this end, the transient voltage stability margin is defined as follows (see reference [22] ):

$$
\xi_{u}=\min \left\{\int_{t}^{t+\tau_{c r}} \frac{u(s)-u_{c r}}{u_{N}-u_{c r}} d s, \quad t \in\left[t_{c l r}, t_{f}\right]\right\}
$$

$t_{c l r}$ is the fault removal time; $t_{f}$ is the end time of the simulation. In $\left[t_{c l r}, t_{f}\right]$, the transient voltage curve is scanned with a time window of width $\tau_{c r}$, as shown in Figure 1.

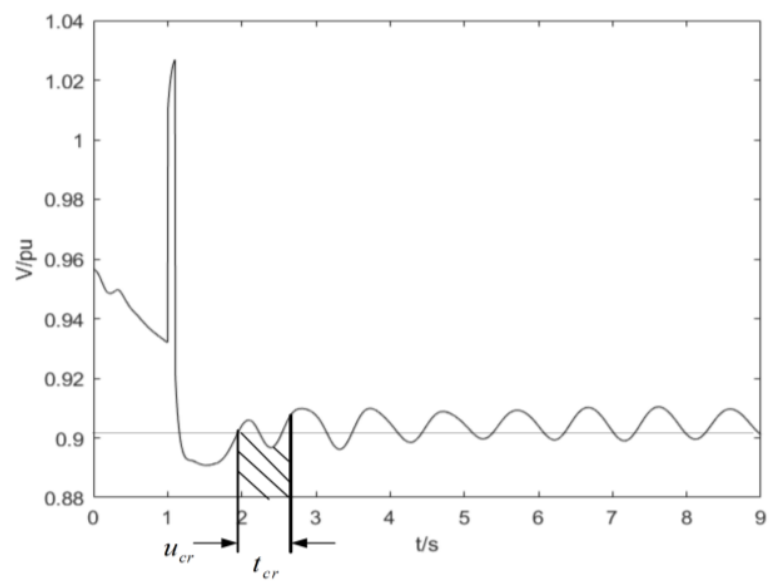

Fig. 1. Transient voltage stability margin.

Among them, the area of the shaded part after the standardization process is the voltage stability margin in the current window. If the voltage in the window is always equal to the rated value, the voltage stability margin is 1 . When the window voltage is always equal to $u_{c r}$, the voltage stability margin is zero. Calculate the voltage stability margin in each scanning window during the entire transient process, and take the minimum value as the system transient voltage stability margin $\xi_{u}$. Since this index is defined on the basis of area, its changes are more continuous and the smoothness is also better.

However, the voltage stabilization accidents in power systems are usually caused by one or more weak buses and then gradually spread to system-wide accidents. So it is a significant task to identify voltage weak buses and implement the corresponding control measures to prevent power systems voltage instability events.

As far as transient voltage stability is concerned, several buses with smaller $\xi_{u}$ are voltage weak buses.

\section{Voltage regulation control strategy with distributed photovoltaic participation}

The installed capacity of new energy in Anhui Province has exploded. In the past three years, the scale of photovoltaic power has increased 8 times, and the scale of wind power has nearly doubled. However, the new energy grid-connected voltage level is low, so the distributed characteristics are obvious. The gridconnected capacity of the province's $110 \mathrm{kV}$ and below voltage levels exceeds $95 \%$ of the province's total capacity, and is dispersed in various voltage-level grids such as $35 \mathrm{kV}, 10 \mathrm{kV}$, and $380 \mathrm{~V}$. And after the Jiquan UHVDC is put into operation, AC and DC failures will cause a large number of distributed photovoltaic disorderly de-grid, further aggravating the voltage stability problem of the power grid in Anhui Province.

In this paper, the main network of Anhui Province is reduced to a three-machine, nine-node model, sketch map of Anhui Province power grid is shown in Figure 2, and according to the $220 \mathrm{kV}$ Taolou substation in Hefei, the equivalent model of distributed photovoltaic intensive access distribution network is established, which can be seen in the Figure 3.

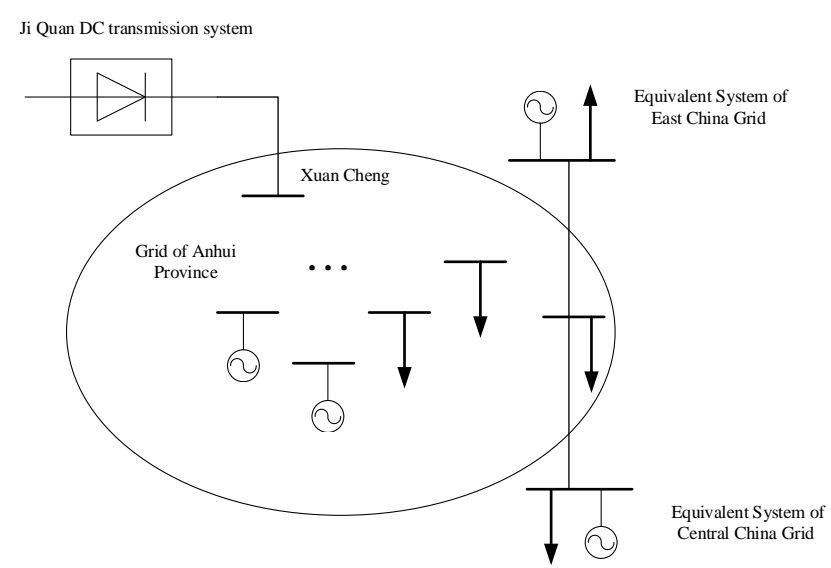

Fig. 2. Sketch map of Anhui Province power grid. 


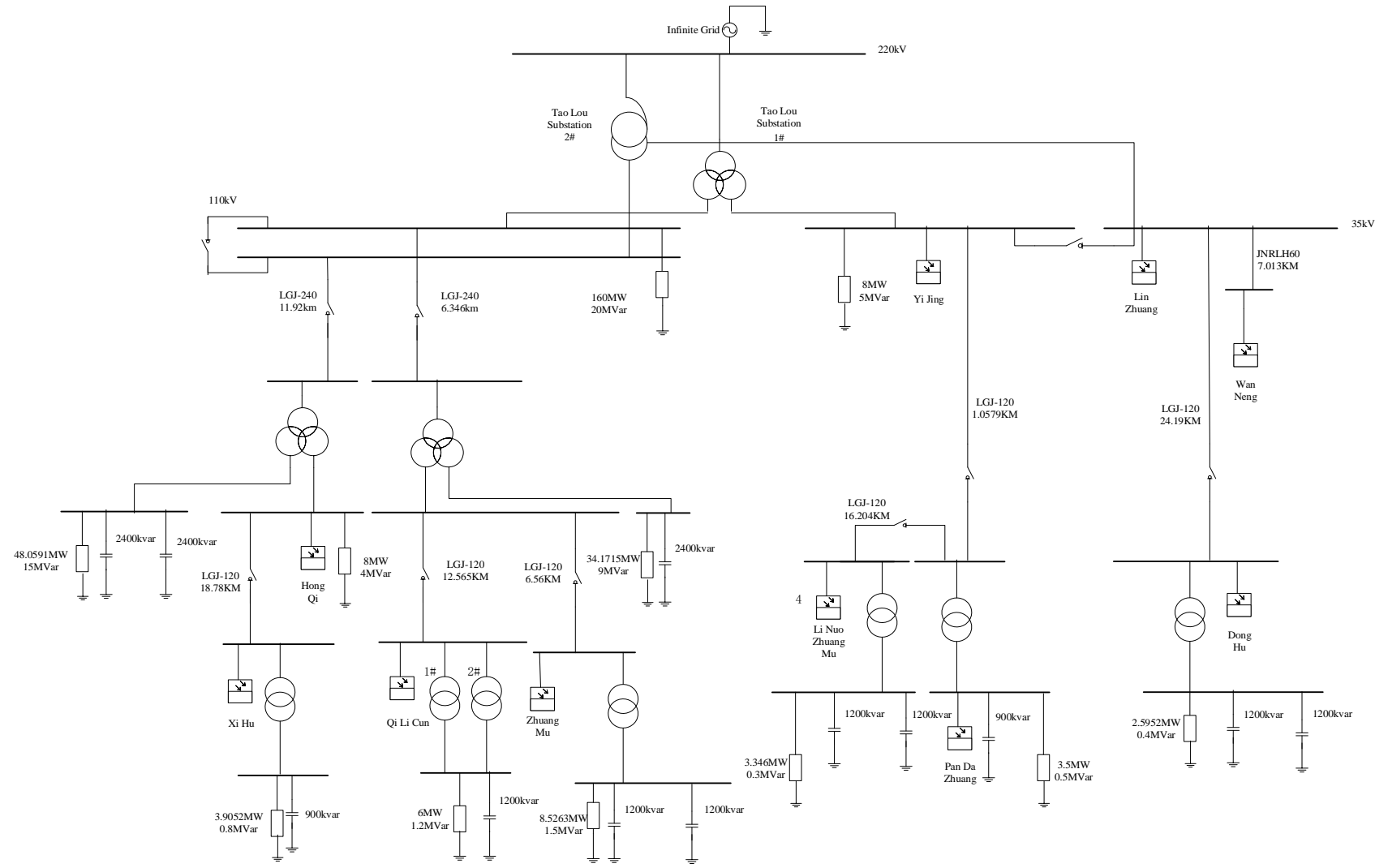

Fig. 3. Topological graph of Taolou Substation.

In the simulation, an integrated load model of induction motor plus constant impedance is used. For detailed parameters of induction motor load(see reference [23] ). There are 10 distributed PV power stations under the Taolou substation, the specific parameters are shown in the table below:

Table 1. Various distributed photovoltaic parameters.

\begin{tabular}{|c|c|c|c|}
\hline Name & $\begin{array}{c}\text { Active power } \\
\text { (MW) }\end{array}$ & $\begin{array}{c}\text { Reactive } \\
\text { power } \\
\text { (MVar) }\end{array}$ & $\begin{array}{c}\text { Maximum } \\
\text { reactive power } \\
\text { that can be } \\
\text { output } \\
\text { (MVar) }\end{array}$ \\
\hline Yi Jing & 35.0 & 0.2 & 19.2 \\
\hline Lin Zhuang & 38.0 & 0.2 & 12.3 \\
\hline $\begin{array}{c}\text { Li Nuo } \\
\text { Zhuang Mu }\end{array}$ & 18.0 & 2.0 & 6.7 \\
\hline Dong Hu & 16.0 & 2.0 & 10.0 \\
\hline Wan Neng & 18.0 & 0.2 & 8.5 \\
\hline Hong Qi & 18.0 & 1.0 & 7.7 \\
\hline Xi Hu & 20.0 & 1.0 & 33.6 \\
\hline
\end{tabular}

\begin{tabular}{|c|c|c|c|}
\hline Qi Li Cun & 30.0 & 2.0 & 24.5 \\
\hline Zhuang Mu & 19.0 & 2.0 & 4.2 \\
\hline $\begin{array}{c}\text { Pan Da } \\
\text { Zhuang }\end{array}$ & 8.0 & 0.1 & 5.9 \\
\hline
\end{tabular}

In view of the noise and other interference factors in the process of actual system signal sampling and transmission, it is necessary to filter the measurement data. The Figure 4 is shown in the process of a distributed $\mathrm{PV}$ voltage control strategy based on the transient voltage margin. Here are the steps:

Step 1: Consider the system's mesh frame structure, reactive compensation, line trend and other factors, determine the key bus set and voltage-time binary table criteria.

Step 2: Measure the bus voltage in real time and filter the processing, when the voltage is below $V_{c r}$, start calculation, over time to calculate the indicator, and compare with the previous calculation and save the small value.

Step 3: When $\xi_{u}<\xi_{u r}$, determine the bus to be a voltage weak bus, and sort bus based on transient voltage margin size. And make distributed photovoltaics near voltage weak buses provide reactive power support. Otherwise, when the voltage is higher than $V_{c r}$, or when the system transient voltage is determined to be stable, the indicator is zeroed and the monitoring is continued. 


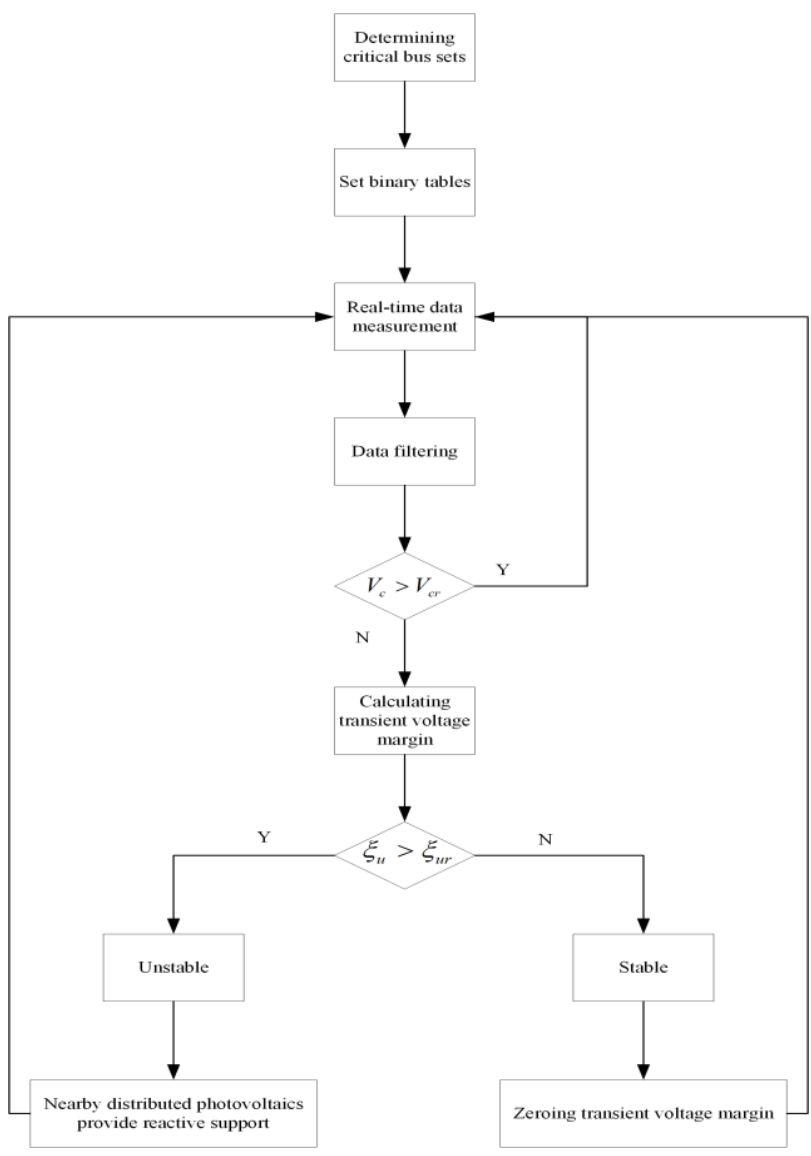

Fig. 4. distributed PV voltage control strategy based on the transient voltage margin.

\section{Simulation}

Table 2 is the simulated operating condition spree when distributed photovoltaics are not involved in regulation

Table 2. Simulation system parameters

\begin{tabular}{|c|c|c|c|}
\hline $\begin{array}{c}\text { Load active } \\
\text { power(MW) }\end{array}$ & $\begin{array}{c}\text { Load reactive } \\
\text { power(MVar) }\end{array}$ & $\begin{array}{c}\text { DC } \\
\text { transmission } \\
\text { power(MW) }\end{array}$ & $\begin{array}{c}\text { Converter } \\
\text { station } \\
\text { reactive } \\
\text { power } \\
\text { compensati } \\
\text { on(MVar) }\end{array}$ \\
\hline 1300 & 60 & 1000 & 500 \\
\hline
\end{tabular}

Set $\mathrm{t}=1 \mathrm{~s}$ DC bipolar blocking, the dynamic response curve of the system voltage is shown in Figure 5. The voltage binary table to be satisfied by the grid is $(0.75 \mathrm{pu}$, 1s). The calculation shows that the transient voltage can be kept stable, but the transient voltage stability margin is low. The 220kv busbar in First Taolou Substation has the lowest transient voltage stability margin of 0.5862 .

If the DC input power is increased, the voltage stability margin of the $220 \mathrm{kv}$ bus in First Taolou Substation will be further reduced. Therefore, it is necessary to take appropriate control measures to improve the system transient voltage stability. The lower limit of the transient voltage stability margin is set to 0.67 , and after the distributed photovoltaics near the $220 \mathrm{kv}$ bus in First Taolou Substation line are compensated in the form of SVG, the system voltage curve is shown in Figure 6.

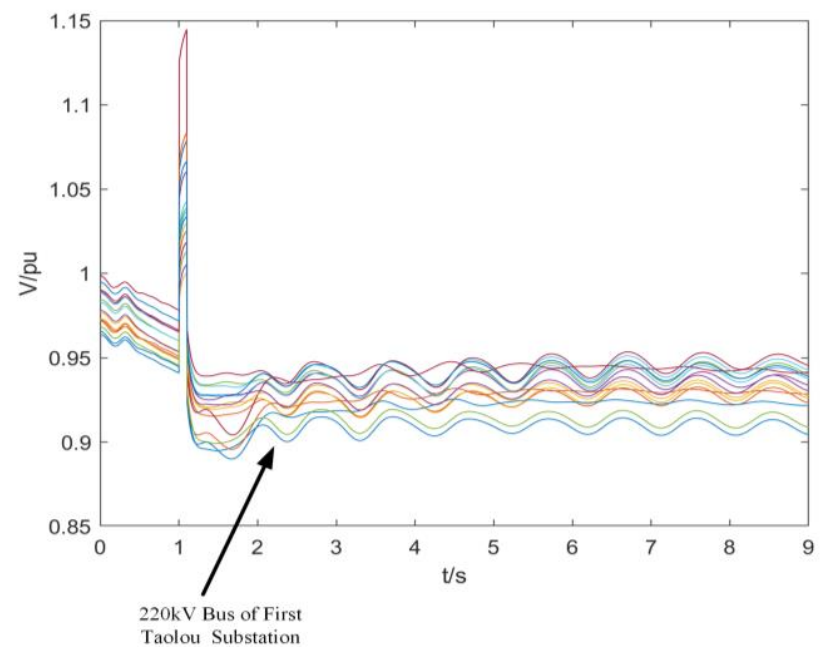

Fig. 5. Voltage response when HVDC line was blocked without control.

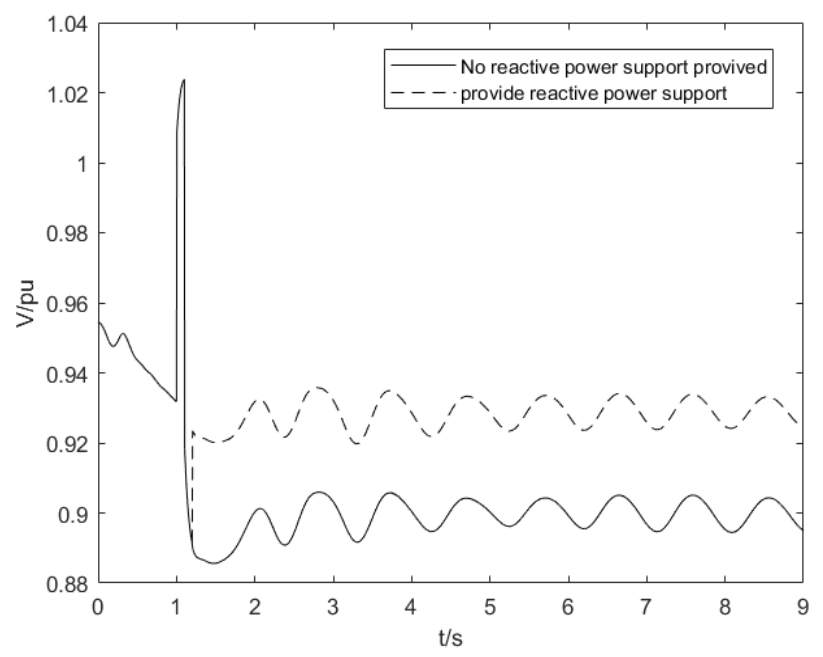

Fig. 6. Voltage of $220 \mathrm{kV}$ bus of first Taolou Substation

It can be seen from Figure 4 that after the distributed photovoltaic system provides reactive power support, the transient stability of the system has been effectively improved. The bus with the lowest transient stability margin has been increased from 0.5862 to 0.6889 , which meets the transient voltage stability requirements.

\section{Conclusion}

This paper calculates the transient voltage margin based on the transient response curve of the receiving end grid after DC blocking, so as to select the weak voltage bus. Utilizing the existing resources of the receiving end grid, the stability margin of the receiving end grid can be improved without large investment. It should be pointed out that the current research does not involve the optimization of photovoltaic reactive power. In addition, the coordination and optimization of transient and steady- 
state or quasi-steady-state emergency control will also be the focus of the next research.

This work was supported by the Science and Technology Project of the State Grid Corporation of China Headquarters "Research and Application of Operation and Control Technology in UHVDC Receiving Power System with High PV Penetration”.

\section{References}

1. Wang Juanjuan, Zhang Yao, Xia Chengjun, PST 12, 30 (2008)

2. Taylor $\mathrm{C} \mathrm{W}$, Power system voltage stability (New York: McGraw-Hill, 1994)

3. Wang Jianming, Sun Huadong, Zhang Jian, PST 12, 66 (2012)

4. Fang Yongjie, AEPS, 4, 1 (2013)

5. Li Kai, Shao Dejun, Xu Youping, Shen Xuhui, Sun Haishun, Zhang Wannan, PST 8, 2961 (2019)

6. Zhang Kaiyu, Cui Yong, Zhuang Kanqin, PSPC 22, 139 (2017)

7. Wang Yating, Zhang Yichi, Zhou Qinyong, PST 1, 22 (2017)

8. Jin Yiding, Yu Zhao, Li Mingjie, PST 7, 2096 (2018)

9. Zheng $\mathrm{Xu}$, Influence and Countermeasures of UHVDC Transmission on Power System Stability (Wuhan University, 2016)

10. Zhao Shuang, Study on STATCOM with Energy Storage to Enhance the Stability in AC/DC Hybrid
Transmission System (Huazhong University of Science and Technology, 2016)

11. Yang Xiongping, Luo Xiangdong, Li Yangxu, Yang Yinguo, PSPC. 22, 40 (2008)

12. Liu Siliang, Huang Wei, Zhang Yongjun, IJSGCE, 8, 580 (2019)

13. Erhab Youssef, Rasha M. El Azab, Amr M. IJSGCE, 4, 226 (2015)

14. Prabha Kundur, Power system stability and control (Beijing: China Electric Power Press, 2001)

15. Xue Yusheng, Quantitative theory of motion stability - analysis of non-autonomous and nonlinear multirigid body system (Jiangsu Science and Technology Press, 1999)

16. DL/T 1234-2013 Technique specification of power system security and stability calculation (China, 2013)

17. Hou Jianlan, Liu Yuquan, Xie Xiaorong, EPAE 10, $151(2015)$

18. Q/CSG 11004-2009, Guide on security and stability analysis for CSG (China, 2009)

19. Zheng Chuancai, Guan Lin, PST 1, 32 (2012)

20. Ashutosh Tiwari,Venkataramana Ajjarapu, IEEE TPS 1, 305(2011)

21. Xu Xin, Zhang Hengxu, Li Changgang. AEPS 18, 143 (2016)

22. Mou Xiaoming, Li Zhimin, PST 8, 163 (2012)

23. Hou Jianlan, Liu Yuquan, Xie Xiaorong, et al. EPAE, 2015, 35(10):151-156. 